\title{
Human Milk Oligosaccharides at the Interface of Maternal-Infant Health
}

\author{
Lars Bode
}

$\mathbf{H}$ UMAN MILK OLIGOSACCHARIDES (HMOs) are a family of structurally diverse, complex sugars abundant in breast milk. ${ }^{1}$ HMO concentrations in human milk often exceed the concentration of all human milk proteins combined (Table 1). HMO profiles can vary based on genetics and geographic location, and can change over the course of lactation., HMOs are resistant to low $\mathrm{pH}$ and intestinal enzymes and are found in the urine and feces of breastfed infants. The oligosaccharides currently added to infant formula are structurally distinct from HMOs; however, some brands are beginning to include certain HMOs in newer formulas.

HMOs act as prebiotics by providing a metabolic substrate for the growth of potentially beneficial bacteria. Bacterial communities in infant feces are able to grow when HMOs are provided as the only source of carbohydrates. Differences in microbial growth were seen with pooled HMO exposure versus individual HMOs; different responses were also seen with structurally different HMOs. The structure-dependent effects of HMOs depend on the infant and mother (e.g., "secretor status" of the mother can alter HMO composition in breast milk and HMO exposure in the infant).

In addition to providing "food for bugs," accumulating evidence suggests that HMOs have protective effects against infectious agents. HMOs have been shown to exhibit unique antimicrobial properties against group B Streptococcus, a leading cause of bacterial infection in newborns (Fig. 1). ${ }^{3}$ The bacteriostatic activity was limited to specific nonsialylated HMOs (i.e., lacto-N-tetraose) and synergistic with other conventional antibiotic agents (i.e., vancomycin and ciprofloxacin). HMOs have also been shown to prevent infections by acting as antiadhesive antibacterials. HMOs isolated from pooled human milk significantly reduced attachment of en-

Table 1. Macronutrient Composition in Milk

\begin{tabular}{lcc}
\hline Macronutrient, $g / L$ & Human milk & Bovine milk \\
\hline Protein & 12 & 35 \\
Fat & 35 & 35 \\
Lactose & 65 & 45 \\
Oligosaccharides & $5-15$ & 0.05 \\
\hline
\end{tabular}

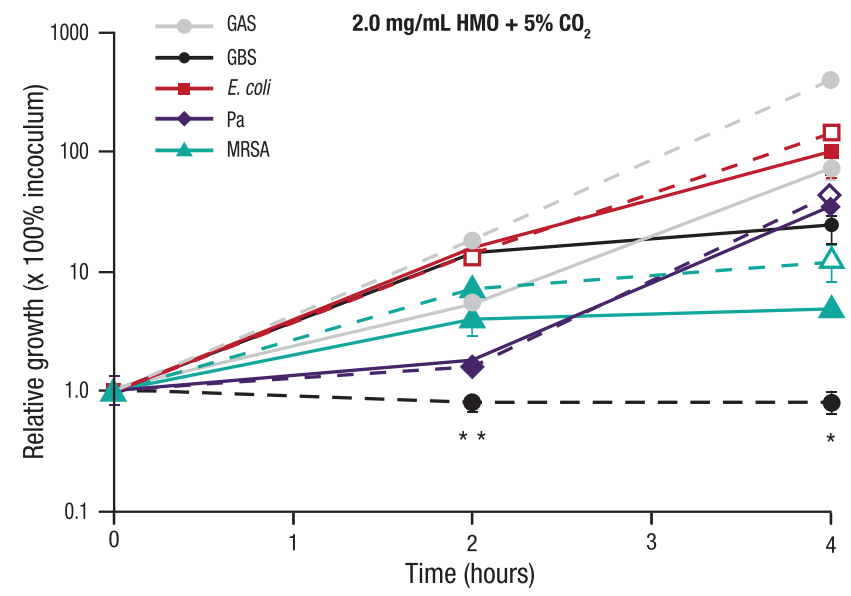

FIG. 1. HMOs inhibit group B Streptococcus growth. ${ }^{3} \mathrm{GAS}$, group A Streptococcus; GBS, group B Streptococcus; HMOs, human milk oligosaccharides; MRSA, methicillin-resistant S. aureus; $\mathrm{Pa}$, Pseudomonas aeruginosa; $\mathrm{pHMO}$, pooled human milk oligosaccharides. Solid line, no pHMO; dotted line, with $\mathrm{pHMO}$.

teropathogenic Escherichia coli (EPEC) to cultured epithelial cells and reduced EPEC colonization in suckling mice compared with untreated controls. ${ }^{4}$ A separate study demonstrated that HMOs reduce attachment and cytotoxicity of the protozoan parasite Entamoeba histolytica when cultured with human intestinal epithelial cell lines. ${ }^{5}$

HMOs may also have indirect effects on the infant microbiome by modulating epithelial and immune cell responses. Pretreatment of bladder epithelial cells with HMOs reduced invasion of uropathogenic E. coli (UPEC) and decreased host cell cytotoxicity. ${ }^{6}$ HMOs also reduced UPECmediated mitogen-activated protein kinase and nuclear factor-kappa B activation, suggesting that HMOs are protective against the proinflammatory effects of UPEC infection. Specific HMOs have been shown to directly affect immune responses by blocking macrophage inflammation; these HMOs are being tested in ongoing studies in animal models of chronic inflammation.

Associate Professor and Larsson-Rosenquist Chair of Collaborative Human Milk Research, Department of Pediatrics and LarssonRosenquist Foundation Mother-Milk-Infant Center of Research Excellence (LRF MoMI CoRE), University of California, San Diego, La Jolla, California. 


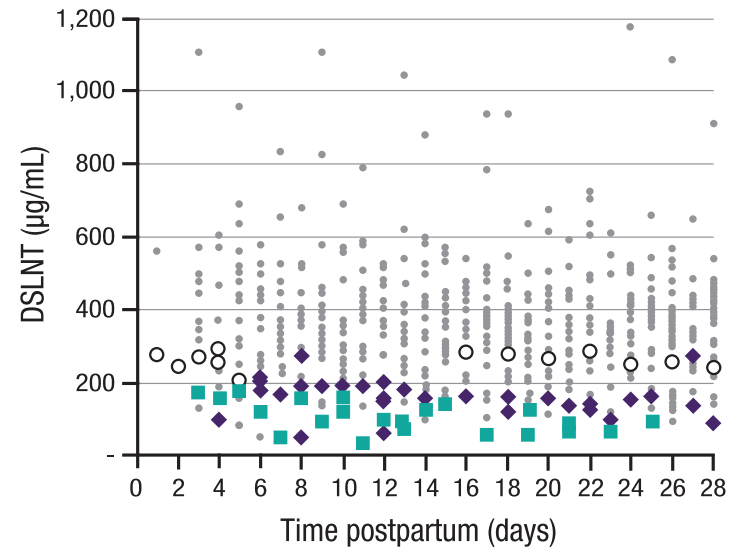

HMOs may play a role in preventing necrotizing enterocolitis (NEC), one of the most common and fatal gastrointestinal disorders in preterm infants. The risk of NEC is $\sim 6$ - to 10-fold higher in formula-fed infants than breastfed infants ${ }^{7}$; HMOs may contribute to the lower incidence of NEC in breastfed infants. To test this hypothesis, rat pups were orally gavaged with formula supplemented with or without pooled HMOs and exposed to hypoxia episodes. ${ }^{8}$ Formula supplemented with HMOs significantly improved 96-hour survival $(95.0 \%$ versus $73.1 \%)$ and reduced NEC pathology scores $(0.44$ versus $1.98 ; p<0.001)$ in neonatal rats compared with formula alone. Further analyses demonstrated that a single HMO, disialyllacto-Ntetraose (DSLNT), had protective effects against NEC. A multicenter clinical cohort study was conducted to assess whether DSLNT can help prevent NEC in preterm infants. ${ }^{9}$ The HMO composition in breast milk from 200 mothers with very low birth weight infants was analyzed for the first 28 days postpartum. Among the eight infants in the cohort who developed NEC (Bell stage 2 or 3), DSLNT concentrations were significantly lower in almost all milk samples than in controls (i.e., infants who did not develop NEC; Fig. 2). Lower concentrations of DSLNT in breast milk could identify NEC cases before onset, suggesting that DSLNT may be a potential noninvasive marker used to identify at-risk infants and screen donor milk. Human intervention studies are needed to better define how DSLNT may prevent preterm infants from developing NEC. The same approach of combining preclinical studies and human cohort studies to inform clinical intervention studies is being applied to other diseases (e.g., environmental enteric dysfunction and diarrheal disease, infant microbiome and obesity, allergies, and asthma).

HMO composition in breast milk is driven by several maternal factors, including genetics and possibly epigenetics, the environment (e.g., diet, lifestyle, and smoking/drug exposure), health and disease, and parity. For example, high-fat diets are associated with less sialylated milk oligosaccharides in humans and mice, and HMO concentration has been shown to change with increase in parity. Additional studies are needed to determine how HMOs and lactation influence maternal health.

\section{References}

1. Bode L. Human milk oligosaccharides: Every baby needs a sugar mama. Glycobiology 2012;22:1147-1162.

2. McGuire MK, Meehan CL, McGuire MA, et al. What's normal? Oligosaccharide concentrations and profiles in milk produced by healthy women vary geographically. Am J Clin Nutr 2017;105:1086-1100.

3. Lin AE, Autran CA, Szyszka A, et al. Human milk oligosaccharides inhibit growth of group B Streptococcus. J Biol Chem 2017;292:11243-11249.

4. Manthey CF, Autran CA, Eckmann L, et al. Human milk oligosaccharides protect against enteropathogenic Escherichia coli attachment in vitro and EPEC colonization in suckling mice. J Pediatr Gastroenterol Nutr 2014;58:165-168.

5. Jantscher-Krenn E, Lauwaet T, Bliss LA, et al. Human milk oligosaccharides reduce Entamoeba histolytica attachment and cytotoxicity in vitro. Br J Nutr 2012;108:1839-1846.

6. Lin AE, Autran CA, Espanola SD, et al. Human milk oligosaccharides protect bladder epithelial cells against uropathogenic Escherichia coli invasion and cytotoxicity. J Infect Dis 2014;209:389-398.

7. Lucas A, Cole TJ. Breast milk and neonatal necrotising enterocolitis. Lancet 1990;336:1519-1523.

8. Jantscher-Krenn E, Zherebtsov M, Nissan C, et al. The human milk oligosaccharide disialyllacto-N-tetraose prevents necrotising enterocolitis in neonatal rats. Gut 2012;61:1417-1425.

9. Autran CA, Kellman BP, Kim JH, et al. Human milk oligosaccharide composition predicts risk of necrotising enterocolitis in preterm infants. Gut 2017 [Epub ahead of print]; DOI: 10.1136/gutjnl-2016-312819.

Address correspondence to: Lars Bode, PhD

Department of Pediatrics and Larsson-Rosenquist Foundation Mother-Milk-Infant Center of Research Excellence (LRF MoMI CoRE) University of California San Diego 9500 Gilman Dr La Jolla, CA 92093

E-mail: lbode@ucsd.edu 